\title{
Caractéristiques sociodémographiques associées à la consommation alimentaire des ménages de Bamako
}

\author{
Sociodemographic characteristics associed with food consumption by households in Bamako
}

Dramé $\mathrm{M}^{1}$, Diarra $\mathrm{S}^{2}$, Sangho $\mathrm{O}^{3,4}$, Diawara $\mathrm{F}^{1,3,4}$, Sangho $\mathrm{F}^{3,4}$, Coulibaly $\mathrm{D}^{1,4}$, Coulibaly $\mathrm{CA}^{1,3}$, Sangho $\mathrm{A}^{4}$, Ag $\mathrm{Iknane}^{1,3,4}$ 1 Institut National de Santé Publique (INSP), Bamako, Mali
2 Système d'Alerte Précoce, Chargé des enquêtes nutritionnelles, Mali
3 Département d'Enseignement et de Recherche en Santé Publique,
FMOS, USTTB, Mali

${ }^{4}$ Faculté de Pharmacie, FAPH, USTTB, Mali

\section{Résumé}

Introduction: La consommation alimentaire est une activité qui engage les individus ainsi que les ménages. La présente étude avait pour objectif d'évaluer les caractéristiques sociodémographiques associées à la consommation alimentaire dans le District de Bamako en février 2016. Matériels et méthodes : Une analyse secondaire des données de d'une enquête nationale de la sécurité alimentaire et nutritionnelle réalisée en février 2016 et a porté sur 1429 ménages du district de Bamako. La régression logistique multinomiale a été utilisée pour le lien entre le profil de consommation alimentaire des ménages avec leurs caractéristiques sociodémographiques. Résultats: Les résultats ont montré que $78,8 \%$ des ménages avaient un score de consommation alimentaire acceptable. Le score de consommation alimentaire était statistiquement associé au sexe, à l'âge, à la situation matrimoniale du chef de ménage, au statut de résidence et au quintile de richesse du ménage. L'analyse de la diversité alimentaire montrait que $77,8 \%$ des ménages avaient un score de diversité alimentaire (SDA) élevée. Le niveau d'instruction et le statut matrimonial étaient les seules variables qui n'avaient pas de lien statistique avec le SDA. Conclusion: La majorité des ménages avait un SCA acceptable. Cependant il y a lieu de sensibiliser pour des bonnes pratiques d'alimentations.

Mots clés: score de consommation alimentaire, caractéristiques, sociodémographiques, ménage, Bamako.

\footnotetext{
Abstract

Introduction: Food consumption is an activity that engages individuals as well as households. The present study aimed to evaluate the socio-demographic characteristics associated with the food consumption of 1,429 households in the District of Bamako in February 2016. Material and methods: A secondary analysis of data from a national food and nutrition security survey carried out in February 2016 which involved 1,429 households in the district of Bamako. Multinomial logistic. regression was used to link the profile of household food consumption with their socio-demographic characteristics. Results: The results shown that $78.8 \%$ of households had an acceptable food consumption score. The food consumption score (FCS) was statistically associated with sex, age, marital status of head of household, residence status and household wealth
}

quintile. The analysis of dietary diversity showed that $77.8 \%$ of households had a high dietary diversity score (HDDS). Literacy and marital status were the only variables that did not have a statistical relationship with HDDS. Conclusion: The majority of households had an acceptable ACS. However, there is reason to raise awareness for good eating practices

Key words: food consumption, socio-demographic characteristics, Bamako.

\section{Introduction :}

La consommation alimentaire est une activité qui engage les individus ainsi que les ménages. Les différences au niveau des individus ou au niveau des ménages occasionnent également des différences des modes de consommation tant au niveau des individus que des ménages. C'est pour cette raison qu'il est nécessaire de bien décrire les traits caractéristiques des ménages ainsi que les profils de leurs membres. La caractérisation des ménages s'avère nécessaire avant d'analyser leur mode de consommation alimentaire (1).

Si les enquêtes de consommation alimentaire étaient jusqu'à présent davantage exploitées en épidémiologie analytique, elles s'inscrivent aujourd'hui de plus en plus dans un objectif de surveillance et d'évaluation des risques alimentaires et nutritionnels (2). Cependant la consommation alimentaire évolue et se diversifie par les plats consommés mais aussi par les modes de consommation (5).

Le régime alimentaire dans les pays Sahéliens n'est pas seulement céréalier, mais repose de plus en plus sur un éventail de produits qui deviennent importants localement et qui augmentent (6). Selon les pays, la consommation de tubercules et racines (manioc, patate douce, igname, pomme de terre), de légumineuses (haricots secs, pois de terre surtout), de viande, de poison, de lait ou de légumes occupe une place importante (6). Par ailleurs, d'autres groupes de produits tendent aussi à émerger. Les produits maraîchers, les fruits et légumes, les légumineuses, les huiles végétales, le poisson, les tubercules occupent la première place dans des pays comme la Guinée-Bissau, le Burkina Faso, le Cap-Vert, le Sénégal et le Mali (6).

$\mathrm{Au}$ Mali, les politiques de Sécurité alimentaire sont restées longtemps fondées principalement sur les objectifs de développement de la production céréalière. Ces politiques se justifiaient par l'importance de la population rurale, centrée sur des stratégies d'autoconsommation familiale ou communautaire, et par l'importance des céréales dans les régimes alimentaires (7).

II existe de très grandes disparités entre les quartiers : les familles aisées ont des conditions de vie comparables 
à celles des pays développés, tandis que les plus défavorisées cherchent à assurer leur survie (8).

La nature et la forme des aliments consommés au Mali, ne différent pas de celles observées dans la plupart des autres pays, compte tenu de la faible diversité des produits disponibles et de la monotonie des modèles de consommation alimentaire (7).

Toutes les familles consomment, au déjeuner et au dîner, des plats ayant une structure commune, associant une base céréalière et une sauce (8). Un certain nombre de facteurs conditionnent les contraintes et ressources de l'Homme pour sa consommation alimentaire. La consommation alimentaire des ménages ne peut donc être analysée indépendamment de l'ensemble des caractéristiques sociodémographiques : Sexe, âge, statut, situation matrimoniale, quintile de richesse, localisation géographique.

Bamako, zone fortement urbanisée où les habitants ont un mode de vie relativement aisé, et aussi une zone de précarité où les habitants vivent dans les conditions moins aisées. C'est sur la base de toutes ces considérations que le choix de la zone a été opéré pour la réalisation de la présente étude dont l'objectif était d'évaluer la consommation alimentaire en lien avec les caractéristiques sociodémographiques des ménages de Bamako en février 2016.

\section{Matériels et méthode}

L'enquête a concerné les données des six communes du district de Bamako (confère carte 1). Chaque commune abrite un centre de santé de référence.

\section{Type et période d'étude}

Une analyse secondaire des données d'une étude transversale descriptive par sondage stratifié en grappe à deux degrés reposant sur les données de l'enquête Nationale de Santé Nutrition (ENSAN) de 2016 (9) au niveau des six communes du district de Bamako a été réalisée. Notre étude, réalisée de décembre 2017 à mars 2018. La taille de l'échantillon était de 1429 ménages dans la base de données nationale de l'enquête SMART de 2017 (9).

Le profil de consommation alimentaire des ménages à savoir score de consommation et score de diversité alimentaire ont été considérés comme variables dépendantes. Les variables indépendantes ont été constituées par les caractéristiques sociodémographiques des ménages (Age, sexe, situation matrimoniale, niveau d'instruction du chef de ménages, le statut de résidence, le quintile de richesse du ménage).

\section{Analyse des données}

Nous avons procédé à une analyse descriptive des caractéristiques sociodémographiques sous forme de tableau. Nous avons réalisé une analyse univariée pour identifier les variables significatives au seuil de 0,05 à mettre dans le modèle de régression multivariée. Pour identifier les facteurs associés à la consommation alimentaire des ménages, des analyses de régression multivariée ont été effectuées. Les indicateurs SCA et SDA ont été traités comme des variables dépendantes. Les variables indépendantes: Age, sexe, situation matrimoniale, niveau d'instruction du chef de ménages et le statut de résidence, le quintile de richesse du ménage ont été transformées pour ces analyses. Les analyses statistiques ont été faites avec le logiciel SPSS 21.

\section{Résultats}

Le tableau I donne les caractéristiques sociodémographique de notre population d'étude. L'âge moyen des Chef de Ménage était d'environ 50 ans. Les plus jeunes chefs de ménages étaient de la commune IV avec 25 ans et les plus âgés en commune III avec 95 ans. Près de $62,6 \%$ des chefs de ménages étaient monogames parmi les $89,2 \%$ de mariés.

La quasi-totalité des chefs de ménages étaient des hommes soit $93 \%$. La quasi-totalité des ménages était en situation de résident dans leurs localités soit $97,8 \%$. II y avait $2,1 \%$ de déplacés et $0,1 \%$ de retourné. Tous les enquêtés des communes III et IV étaient des résidents. Ceux des communes I, II, V et $\mathrm{VI}$ étaient respectivement de $96,7 \%, 97,4 \%, 98,7 \%$ et $96,6 \%$. La proportion de chefs de ménages sans aucun niveau d'instruction était de $26,2 \%$. Le revenu total moyen habituel s'élevait à 134,185 FCFA avec un écart type de $\pm 170,311$ FCFA. La taille du ménage avait une influence significative sur la consommation alimentaire. La consommation alimentaire est dépendante du niveau de richesse. Les céréales représentaient le principal aliment chez tous les ménages pauvres, moyens et riches. La bouillie était le petit déjeuner de plus de $70 \%$ des ménages à Bamako. Les œufs n'étaient consommés que dans les familles riches. La plupart $(65 \%)$ des différentes classes du quintile consommaient au déjeuner un plat unique, le riz à la sauce.

A Bamako, le score de consommation alimentaire (SCA) des 1429 ménages était acceptable à $78,8 \%$. Les ménages ayant la consommation alimentaire la plus pauvre ou limite prédominaient en commune $\mathrm{VI}$ avec respectivement $15,3 \%$ et $31,4 \%$. Les caractéristiques socio démographiques: sexe, âge, statut, situation matrimoniale des chefs de ménage et quintile de richesse des ménages de Bamako avaient un lien statistique avec le SCA (Tableau II).

Les ménages de Bamako avaient un score de diversité alimentaire (SDA) élevée de 77,8\%. L'étude de la diversité alimentaire en relation avec des caractéristiques sociodémographiques a montré que celle-ci était liée au sexe du chef de ménage, à l'âge, à la situation matrimoniale et au quintile de richesse (Tableau III).

\section{Discussion \\ Age}

La tranche d'âge la plus touchée ayant une alimentation adéquate et diversifié était celle des plus de 45 ans. 
Cette situation s'expliquerait par le fait que cette classe d'âge serait mieux entretenue sur le plan alimentation que les moins de 45 ans.

\section{Le sexe :}

Les hommes étaient les chefs de ménages les plus nombreux. Ce résultat reflète le contexte malien de gestion de la famille qui est même prévue dans le code du mariage où l'homme est le chef de ménage et supporte principalement les charges. Au niveau national en février 2016, 92,8\% des ménages étaient dirigés par des hommes contre $92,9 \%$ en février 2017 (9) cependant, les femmes chefs de ménage avaient plus de chance d'avoir une alimentation adéquate que les hommes. Ceci pourrait s'expliquer par le fait que les femmes s'alimentaient en dehors des repas familiaux.

Statut Matrimonial :

Près de $90 \%$ de nos enquêtés étaient mariés. Cette fréquence était de $93 \%$ en 2016 (10) contre 92,9\% en 2017 (9). Les non mariés avaient plus de chance d'avoir une alimentation adéquate comparés aux mariés.

\section{Statut de résidence :}

Les non-résidents à Bamako avaient plus de chance d'avoir une alimentation adéquate par rapport aux résidents. Ceci pourrait s'expliquer par les dons des ONG, de l'Etat, des agences des Nations Unies et des familles. En effet, c'était des déplacés suite aux différents conflits au nord et centre du pays et des retournés suite aux refoulements de maliens de leurs pays émigrations.

Niveau d'instruction :

Plus d'un quart des chefs de ménages n'avaient aucun niveau d'instruction. Cette fréquence était très faible, comparée aux 46,5\% de 2016 (10) et aux 52,5\% de 2017 (9). Les instruits étaient plus aptes à avoir une alimentation adéquate par rapport aux non instruits.

Revenu total habituel :

Le revenu total moyen habituel d'environ 135 FCFA était fortement corrélé à la consommation alimentaire du ménage.

Score de consommation alimentaire (SCA) et score de diversité alimentaire (SDA) :

L'étude a révélé que $78,8 \%$ des ménages avaient un score de consommation acceptable. Ce score était supérieur à celui trouvé par l'ENSAN Mali en Février 2017 avec 69,4\% acceptable et inférieur à celui trouvé par ENSAS en juin 2016 au Sénégal (11) qui montre que $83,1 \%$ des ménages Sénégalais ont une consommation alimentaire acceptable. II y a une légère diminution de la consommation alimentaire entre les deux éditions de 2016 (10) et de 2017 (9) au Mali. Cette diminution pourrait être due à l'insécurité alimentaire et sociale, aux mouvements de population, à l'accès insuffisant aux marchés (hausse des prix et insuffisance de pouvoir d'achat), à la baisse de l'assistance alimentaire.

Dans notre étude $97,2 \%$ des ménages ont consommé plus de quatre groupes d'aliments la veille de l'enquête d'où un score de diversification élevé. Ce score était inférieur à celui trouvé par l'ENSAN avec 94,2\% des ménages en Septembre 2015 ayant consommé plus de quatre groupes d'aliments. Cette amélioration du score pourrait s'expliquer par le fait que l'enquête s'est déroulée à une période proche de la fête de tabaski. Lors de cette fête, la consommation alimentaire augmente considérable dans tous les ménages. Elle est essentiellement basé sur la viande avec les abattages de montons ou de bœufs ou autres animaux. Les familles préparent abondamment et reçoivent aussi des aliments en cadeaux soit par filiation soit par alliance.

La prépondérance du modèle alimentaire de Bamako était basée sur les céréales. Plus de $90 \%$ des ménages interrogés consommaient les céréales. La faible consommation de fruits et légumes figure parmi les dix principaux facteurs de risque de mortalité, selon l'OMS. Or, les œufs et les fruits comme la banane, les mangues ne sont pas aussi coûteux que les protéines animales ou les sucres (bières, jus, etc.) et huiles dont la consommation augmente les dépenses.

\section{Conclusion}

La majorité des ménages avait un SCA acceptable. Le niveau d'instruction avait une influence positive sur le régime alimentaire. La consommation alimentaire était fortement corrélée au revenu du ménage. II y a lieu de renforcer des activités de communication pour le changement de comportement à l'endroit des chefs de ménages sur les bonnes pratiques d'alimentation.

\section{References}

1. MUTEBA Kalala Damien. CARACTERISATION DES MODES DE CONSOMMATION ALIMENTAIRE DES MENAGES A KINSHASA: "ANALYSE DES INTERRELATIONS ENTRE MODES DE VIE ET HABITUDES ALIMENTAIRES ». Thèse de doctorat: Agronomie : Faculté des Sciences agronomiques et Ingénierie biologique, Université de liège-Gembloux agro-bio Tech: Liège: $2014 ; 199$

2. Haut Comité de la Santé Publique. Pour une politique nutritionnelle de santé publique en France. Enjeux et propositions. ENSP. Paris; 2000, p 67.

3. FAO, FIDA et PAM. (2014). L'État de l'insécurité dans le monde 2014. Créer un environnement plus propice à la sécurité alimentaire et à la nutrition. Rome : FAO, 57 pages. http://www.fao.org/3/a-i4030f.pdf.

4. FAO, FIDA et PAM. (2012). L'état de l'insécurité alimentaire dans le monde 2012. La Croissance économique est nécessaire mais elle n'est pas suffisante pour accélérer la réduction de la faim et de la malnutrition. Rome: FAO, 69 pages. http://www.fao.org/ docrep/017/i3027f/i3027f.pdf.

5. Ag Bendech M., Chauliac M., Malvy D. Variabilité des pratiques alimentaires à domicile des familles vivant à Bamako selon le niveau socio-économique. Cahiers Santé, 1996b;6 : 285-97. 
6. Normes de consommation des principaux produits alimentaires dans les pays du CILSS, Juillet 2004, pp.3744.

7. Stratégie nationale de sécurité alimentaire du Mali. Note de synthèse sur la stratégie nationale de sécurité alimentaire au Mali page 161

8. Ag Bendech M ., Chauliac M., Gerbouin Rérolle P., Kanté N., Malvy D.J.M.

Les enjeux de la consommation alimentaire en milieu urbain à Bamako. Santé publique 2000, volume 12, no 1 , pp. $45-63$

9. Horman D. (2004). Chicken connection. Le poulet africain étouffé par l'Europe. Agrobusiness, dumping, souveraineté alimentaire. Bruxelles : Groupe de recherche pour une stratégie économique alternative (GRESEA).

10. Rapport de mission des fora dans les régions et dans le district de Bamako

15/02 au 03/03/2010. Forum sur la nutrition de Bamako, $\mathrm{p} \mathrm{n}^{\circ} 178-179,212 \mathrm{P}$

9. Institut National de la Statistique (INSTAT). Enquête Nationale Nutritionnelle Anthropométrique et de Mortalité rétrospective suivant la méthodologie SMART, Mali 2017 [Internet]. Bamako: INSTAT; 2017 [cité 11 mai 2019] p. 131. Disponible sur: http://www.instatmali.org/contenu/eq/rafsmart17 eq.pdf.

10. Institut National de la Statistique (INSTAT). Enquête Nutritionnelle Anthropométrique et de Mortalité rétrospective, Mali, SMART 2016 [Internet]. Bamako: INSTAT; 2016 juill [cité 11 mai 2019] p. 117. Disponible sur: $\quad$ http://www.instatmali.org/contenu/eq/rafsmart16_eq.pdf

11. Ministère de la Santé et de l'Action Sociale, Direction de la Santé de Réproduction et de la Survie de l'Enfant,
Division de l'Alimentation et de la Nutrition. Enquête Nutritionnelle Nationale utilisant la méthodologie SMART [Internet]. Sénégal: Ministère de la Santé et de l'Action Sociale; 2016 juin [cité 15 mai 2019] p. 188. Disponible sur:

https://www.unicef.org/senegal/french/Rapport_SMART.p df

Liste des tableaux et figure :

Tableau II : Régression multinomiale des variables de caractéristiques sociodémographiques par rapport au score de consommation alimentaire

\begin{tabular}{lcc}
\hline Caractéristiques & $\mathbf{O R}[\mathbf{I C 9 5} \%]$ & $\mathbf{p}$ \\
\hline Sexe du chef de ménage & $1,072[1,04 ; 1,105]$ & 0,0001 \\
Age & $2,434[2,148 ; 2,758]$ & 0,0001 \\
Résidence & $3,034[2,738 ; 3,361]$ & 0,0001 \\
Statut matrimonial & $0,972[0,943 ; 1,002]$ & 0,0001 \\
Niveau d'instruction & $6,405[5,846 ; 7,018]$ & 0,0690 \\
SDA (4groupes & $3,315[3,26 ; 3,371]$ & 0,0001 \\
d'aliments) & & \\
Classe (Pauvre Moyen & $1,072[1,04 ; 1,105]$ & 0,0001 \\
Riche) & \\
\hline
\end{tabular}

Tableau III : Régression multinomiale des variables de caractéristiques sociodémographiques par rapport au score de diversité alimentaire

\begin{tabular}{lcc}
\hline Caractéristiques & OR [IC95\%] & $\mathbf{p}$ \\
\hline Sexe du chef de ménage & $4,592[4,087 ; 5,16]$ & 0,0001 \\
Age & $0,375[0,353 ; 0,398]$ & 0,0001 \\
Résidence & $0[0 ; 0]$ & 0,9800 \\
Statut matrimonial & $1,321[1,161 ; 1,504]$ & 0,0001 \\
Niveau d'instruction & $1,005[0,949 ; 1,065]$ & 0,8600 \\
Classe (Pauvre Moyen & $0,406[0,393 ; 0,42]$ & 0,0001 \\
Riche) & & \\
\hline
\end{tabular}




\section{MALI SANTE PUBLIQUE 2019 SOMASAP}

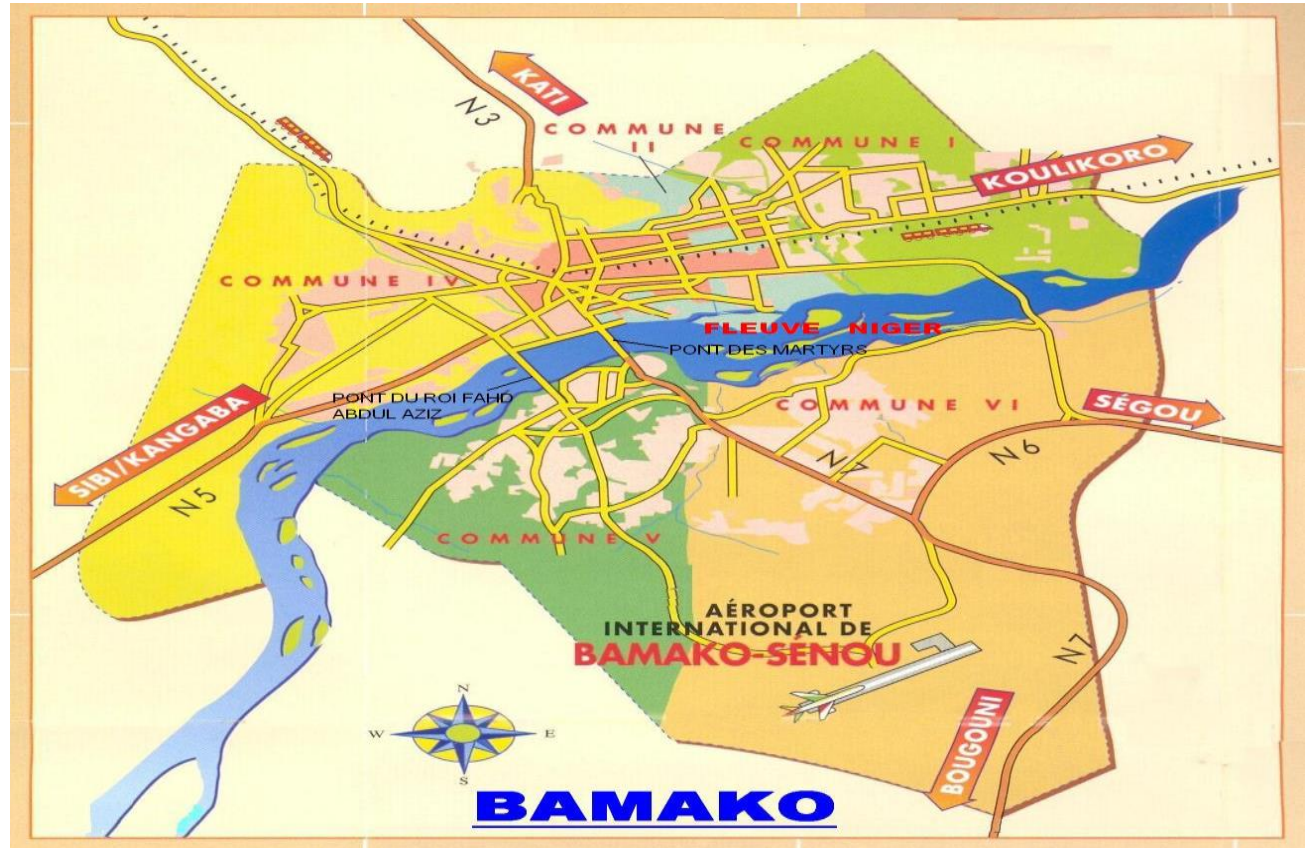

Carte 1 : géographique-Bamako-Map.jpg

Tableau I:

\begin{tabular}{|c|c|c|c|c|c|}
\hline Ages des chefs de ménages & Minimum & Moyenne & Maximum & Ecart Type & \\
\hline Commune I & 26 & 48,69 & 80 & \pm 12 & \\
\hline Commune II & 30 & 49,61 & 85 & \pm 12 & \\
\hline Commune III & 26 & 53,37 & 95 & \pm 13 & \\
\hline Commune IV & 25 & 48,92 & 86 & \pm 14 & \\
\hline Commune V & 26 & 48,4 & 85 & \pm 12 & \\
\hline Commune VI & 26 & 46,08 & 81 & \pm 12 & \\
\hline Total & 25 & 50,14 & 95 & & \\
\hline $\begin{array}{l}\text { Nombre de personnes par } \\
\text { ménage }\end{array}$ & Minimum & Moyenne & Maximum & & \\
\hline Commune I & 48 & 14,05 & 3 & & \\
\hline Commune II & 54 & 11,87 & 3 & & \\
\hline Commune III & 50 & 15,45 & 3 & & \\
\hline Commune IV & 38 & 10,69 & 3 & & \\
\hline Commune V & 56 & 13,68 & 3 & & \\
\hline Commune VI & 36 & 11,17 & 3 & & \\
\hline Total & 56 & 12,82 & 3 & & \\
\hline Statut matrimonial & Monogame (\%) & Polygame (\%) & Non marié (\%) & & \\
\hline Commune I & 54,1 & 38,9 & 7,0 & & \\
\hline Commune II & 64,0 & 25,2 & 10,8 & & \\
\hline Commune III & 65,6 & 21,5 & 13,0 & & \\
\hline Commune IV & 77,0 & 16,7 & 6,3 & & \\
\hline Commune V & 63,6 & 33,9 & 2,5 & & \\
\hline Commune VI & 62,9 & 26,3 & 10,8 & & \\
\hline Total & 62,6 & 30,2 & 7,2 & & \\
\hline Quintile de richesse & Plus pauvre (\%) & Pauvre (\%) & Moyen (\%) & Riche (\%) & Plus riche (\%) \\
\hline Commune I & 0,9 & 4,0 & 9,6 & 23,6 & 61,8 \\
\hline Commune II & 0,9 & 3,0 & 6,1 & 24,2 & 65,7 \\
\hline Commune III & 0,8 & 6,7 & 16,0 & 20,1 & 56,3 \\
\hline Commune IV & 0,0 & 9,5 & 6,9 & 27,3 & 56,3 \\
\hline Commune V & 2,4 & 6,1 & 8,6 & 27,9 & 54,9 \\
\hline Commune VI & 81,2 & 13,9 & 2,5 & 1,2 & 1,3 \\
\hline Total & 24,3 & 8,1 & 7,2 & 18,6 & 41,9 \\
\hline
\end{tabular}

